\title{
Improvement of Work Efficiency for Desk Work Using Biological Information
}

\author{
Shinnosuke Date, ${ }^{1 *}$ Yuta Miyake, ${ }^{2}$ and Takeshi Iwamoto ${ }^{3}$ \\ ${ }^{1}$ Information Systems Engineering, Graduate School of Engineering, Toyama Prefectural University, \\ 5180 Kurokawa, Imizu-shi, Toyama 939-0398, Japan \\ ${ }^{2}$ DOCOMO Technology, Inc., 1-8-1 Akasaka, Minato-ku, Tokyo 107-0052, Japan \\ ${ }^{3}$ Department of Electrical and Computer Engineering, Toyama Prefectural University, \\ 5180 Kurokawa, Imizu-shi, Toyama 939-0398, Japan
}

(Received August 23, 2019; accepted October 4, 2019)

Keywords: IoT, desk work, biological information, interruption

In recent years, long work hours and overtime work have become a social problem in Japan. Therefore, attention is focused on improving the work style in Japan. In particular, there is a demand for improving the efficiency of desk work. In this paper, we aim to clarify the relationship between work and concentration, and the influence of interruptions on the concentration state and the number of working hours (work time) to realize appropriate time allocation in desk work. In the experiment, staff members of the Toyama prefectural office were recruited as subjects to obtain data on the work times and concentration state. We use a device called Spire to determine the concentration state. Spire can acquire respiration information. As a result, it was found to be necessary to continue work for $10 \mathrm{~min}$ or more in order to achieve high concentration and work efficiency. However, it was found that if concentration was not attained within 10 min from the start of work, it was necessary to interrupt the work. It was also found that interruption did not affect the work time or concentration state.

\section{Introduction}

Some countries undergoing economic growth have the problem of overwork. To cope with this problem, the Japanese government is encouraging changes in work style by introducing several policies. ${ }^{(1)}$ People are beginning to pay attention to the working style because of these government policies. The Survey of the Labor Policy Research and Training Organization of the Independent Administrative Agency shows ${ }^{(2)}$ that approximately $20 \%$ of all workers work at their desks. Therefore, improving the efficiency of desk work is becoming important. To improve the work efficiency of desk work, it is desirable to carry out work while maintaining a high concentration.

On the basis of this background, it is necessary to prevent interruptions to concentration due to, for example, task switching as much as possible. In fact, the Toyama prefectural government is aiming to prevent interruptions to concentration by presenting a signboard that shows "no interruption time". There are other signboards that indicate the situation. This signboard

*Corresponding author: e-mail: s-date@puc.pu-toyama.ac.jp

https://doi.org/10.18494/SAM.2020.2559 
can prevent interruptions to concentration. However, the presentation of such information depends on the individual's taste, and it is not always possible for the individual to concentrate during the specified time. In addition, it is considered desirable that workers do not present the signboard if their concentration declines and the interruption does not affect their concentration. Consequently, when presenting "interruptible time" similarly to the above example, it is necessary to estimate the concentration state of workers and to present information dynamically. In addition, various tasks exist in desk work. In order to maintain a high concentration, it is important to plan the order in which to perform these tasks. For example, it may be desirable to start from an earlier task before concentration becomes high and to perform work requiring a high concentration and the longest duration of concentration. However, as with the above example, this plan is executed on the basis of individual judgment and does not necessarily guarantee high work efficiency. Therefore, we need to quantify the relationship between work efficiency and concentration. In particular, if the time when concentration becomes high for each task can be clarified, it should be possible to present information tailored to each task. For example, if it has been identified that "this work requires $20 \mathrm{~min}$ of concentration", it is necessary to reserve a time of $20 \mathrm{~min}$ or more to complete the work. On the basis of this result, it will be possible to devise a plan of work and to present the "time to interrupt", similarly to the above example, according to the work. In this paper, we clarify the relationship between work efficiency and concentration in order to determine the appropriate time allocation for carrying out desk work.

The main contributions of this paper are as follows.

1) Appropriate time allocation of work focusing on concentration

There have been many studies on the estimation of concentration during work. However, to the best of our knowledge, there are no reports on performing tasks with appropriate time allocation in desk work. In this paper, we report the relationship between the concentration state and the number of tasks. In addition, we report the time for which the concentration state in work can be maintained. Our results show that one task should be continued for more than $10 \mathrm{~min}$ in order to complete the task with appropriate time allocation focusing on the concentration state. Furthermore, if concentration is not achieved within $10 \mathrm{~min}$, the task should be interrupted immediately.

2) Relationship between concentration state and interruption

This paper shows that interruptions in desk work have no effect on concentration. Generally, we assume that interruptions during desk work affect concentration; however, in this experiment, we found no clear influence of interruption on concentration.

3) Interruptions are considered to affect business operations. Therefore, it is necessary to consider the influence of interruptions within the appropriate time allocation of work focusing on the concentration state. We investigated the impact on concentration when an interruption occurs in an actual field. As a result, in this experimental environment, interruptions were not found to affect the concentration state. This result disprove the existing notion that "interruption interferes with business."

4) This method can cover many tasks in desk work. Desk work involves various tasks. Research on the concentration state during desk work often focuses on one task. The method of this study can cover most tasks in desk work. 
The remainder of the paper is organized as follows. Related studies are presented in Sect. 2. The experimental environment, experimental methods, task, and interruptions are described in Sect. 3. In Sect. 4, we describe the sensing method in the experiment and the results of the experiment in Sect. 5. The experimental considerations are described in Sect. 6, and the summary of the paper is described in Sect. 7.

\section{Related Works}

This section describes the existing research related to this study. First, the outline of the existing research is described focusing on the estimation method of worker's concentration state in desk work. Next, we outline the existing research focusing on interruptions to work. Finally, the subject of this study is clarified.

\subsection{Outline of existing research on estimation of worker's concentration state}

There have been many studies on the estimation of the concentration state of workers performing desk work. For the estimation of the concentration state, research has been conducted under the assumption that the subject is sitting in a chair and performing tasks such as PC work, study, and office work. ${ }^{(3,4)}$ In particular, Okubo and Fujimura ${ }^{(3)}$ evaluated systems and graphical user interfaces (GUIs) that estimate the degree of concentration from information obtained via an acceleration sensor that is attached to a chair and presents the information to the user. As a result, a strong negative feedback is confirmed between the power spectrum obtained by the Fourier transformation of the acceleration in the direction of three axes and the number of keystrokes on the keyboard. In the evaluation of GUIs, the result is that an interface with graphs and level meters is easier to understand than an avatar alone for studies on the concentration state.

For the purpose of realizing a virtual office in a work-at-home environment, Honda et al. ${ }^{(5)}$ changed the work space in accordance with the degree of concentration. The results of experiments on the degree of concentration confirmed that the change in behavior and the degree of concentration determined from the videos are roughly consistent.

Concentration estimation using various methods has been reported, and it is not limited to desk work. Yajima et al. ${ }^{(6)}$ estimated concentration using body temperature, galvanic skin response (GSR), and pulse rate. They developed the hardware to obtain these biological parameters. As a result, they reported that GSR changes proportionally to the degree of concentration. However, they consider that the accuracy of temperature- and pulse-based concentration estimation must be improved.

There is a device that can estimate the concentration state by measuring eye blinks. Uema et al. ${ }^{(7)}$ estimated the concentration state using JINS MEME, which is a glasses-type wearable device. This device acquires the eye blink speed, the eye blink strength, and the number of blinks. Uema et al. also found that the number of eye blinks decreases when people are concentrated. 


\subsection{Overview of existing research focusing on interruption}

Hashimoto et al. ${ }^{(8)}$ proposed a method of estimating the appropriate timing of interruption in real time from PC operation and speech. As a result, it was confirmed that the accuracy of interrupt estimation is improved compared with previous research by detecting the presence and end of conversation.

In addition, Mark et al. ${ }^{(9)}$ conducted experiments by creating three types of situations to confirm the impact caused by the interruption of operations. Specifically, they conducted an experiment in which they used email correspondence related to human resources under three conditions, namely, no interruption of the task, interruption with a content related to the task, and interruption with a content not related to the task. They performed NASA task load index (NASA-TLX) to evaluate the mental load subjectively after the experiment. As a result, although there were no changes in the number of typographical errors or mistakes regarding the use of honorifics, it was confirmed that the stress and workload increased upon interrupting the task for $20 \mathrm{~min}$. There have also been studies that have confirmed that the state of health improves as a result of interruption and taking rest from the viewpoint of workers' health. ${ }^{(10,11)}$

\subsection{Issues of existing research and positioning of this research}

The degree of concentration estimated from the sensor values output by PC peripheral devices, such as keyboards and computer mice, indicates that the behavior of workers is greatly restricted. Although there is no problem with desk work in the existing research, the desk work handled in this research is wide-ranging, and a method of estimating the degree of concentration independent of the environment is required. In addition, in electroencephalography measurements, the load on workers can be considered. Therefore, in this research, we will estimate the degree of concentration using a wearable device that does not depend on the environment and does not place a burden on the worker.

In addition, in the research focusing on the interruptions of work, the method of estimating the degree of interruption and its influence on the work are described. On the other hand, there are also studies that mention the merit of the improved health status due to interruption. The purpose of this research is to increase work efficiency focusing on the concentration state. Therefore, it is necessary to clarify the influence of interruptions on the concentration state during a task and to consider ways in which task interruptions occur.

\section{Methodology}

In this section, we describe the experimental environment, tasks, interruptions, and experimental method considered in this research. Specifically, the methods of estimating the concentration state and the number of working hours (work time), and confirming interruption are described. 


\subsection{Purpose of experiment}

We aim to clarify the relationship between work efficiency and concentration stake, and the influence of interruptions on the concentration state and working hours. Therefore, we verify the following:

1) relationship between the concentration state and the number of tasks,

2) time of maintaining concentration in work,

3) change to the concentration state after interruption.

The appropriate time allocation in desk work focusing on the concentration state is derived from the examination of 1) and 2). In addition, we clarify the influence of interruptions on the concentration state by examining 3).

\subsection{Experimental environment}

The experimental environment in this study was established with the cooperation of the Toyama Prefectural Government Office for Measures against Decline in Fertility and Citizens' Activity Division, which is promoting the reformation of work styles and active participation of women in society by companies in Toyama Prefecture. In addition, the subjects are four of 20 members. Written informed consent to participate was obtained after explaining the contents of the experiment in advance to the participants. This study was approved by the Ethics Review Board of Toyama Prefectural University.

\subsection{Definition of interruptions}

The interruptions of work in this research are defined as the situations where it is necessary to interrupt desk work in the following cases:

1) The subject receives a call during the task.

2) The subject is spoken to during the task.

As described above, instead of the workers' own actions, situations where their work is interrupted by a third party are defined as interruptions in desk work. In addition, changes in work by the workers themselves, such as voluntarily leaving their seat and meetings, are not treated as interruptions.

\subsection{Definition of task in desk work}

We conducted interviews with the staff and classified the tasks to be tested. In addition, in this research, desk work duties are assumed to include tasks other than PC tasks. As a result of interviews, the tasks have been classified into the following: 
1) email correspondence,

2) what to investigate,

3) document preparation,

4) telephone call,

5) meeting,

6) leaving,

7) going out,

8) document perusal.

In addition to tasks other than the above, the subject may take a break or spend time thinking during actual work hours. Resting and thinking basically do not involve physical tasks. Therefore, it is difficult for a third person to determine whether the person is resting or thinking depending on the worker himself. Therefore, in this experiment, while sitting at a desk for PC work and document entry, the time when no physical work is performed is treated as resting and thinking.

Furthermore, in desk work, since a task is selected on the basis of the judgment of the worker, the definition of the continuation time of the task in the experiment has not been established. Therefore, in this experiment, the state where a task is continued for one minute or more is defined as one task in this experiment. In addition, 4) a telephone call is a task as well as an interruption. In this case, it is considered to be a task (telephone call) if it lasts for more than one minute. On the other hand, if it lasts for less than one minute, it is treated as one interruption (telephone call) occurring during a task. Furthermore, we will treat resting and thinking in the same way as interruptions.

In the experiment, the work time of 4 subjects and the concentration state during that time are acquired. The subjects perform a total of 7 hours of work from 9:00 to 17:00 (except for a $1 \mathrm{~h}$ lunch break). The experimental period is 5 days. We expected that this experiment could result in different outcomes depending on the peak period of the subjects' work. Therefore, this experiment was conducted when the subjects were not busy. We analyzed the acquired data focusing on the relationship between work hours and the concentration state.

Moreover, we consider that there are some tasks that are performed in parallel, such as doing other tasks during a meeting. People sometimes are required to implement several tasks at the same time at the work place. However, in this study, we conducted experiments and studied on the premise that a subject is required to focus on one task as a basic study. We will conduct further research on efficiency of doing several tasks at the same time after analyzing the results of this study and clarifying the relationship between the concentration state and interruptions.

\section{Sensing}

In this section, we describe the method of sensing tasks and concentration state.

\subsection{Method of work estimating time}

We developed a task recording application to identify two points of the task content that subjects performed and the ease of concentration for each task. In the experiment, we assumed 
that this application will be performed in parallel with normal work. Therefore, this application must be a simple operation that interrupts normal work as little as possible. The specifications of the business record application are summarized below. Figure 1 shows the process diagram of the business application.

\subsection{Estimation of concentration state}

In this research, we acquired biological information via a wearable device and estimate the concentration state. Regarding the relationship between the concentration state and biological information, Vlemincx et al. ${ }^{(12)}$ clarified the relationship between task properties and respiratory rate. In their experiment, subjects were asked to perform stressed memory tasks (load tasks) and mental tasks that require concentration (central tasks). As a result, the following results are obtained relative to the normal respiratory rate.

Normal respiratory rate $<$ respiratory rate for central tasks $<$ respiratory rate for load tasks

From the above, the respiratory rate is considered to be useful as biological information for estimating the concentration state. Therefore, in this study, the concentration state is estimated by acquiring the respiratory rate. In the experiment, we use a wearable device called Spire, ${ }^{(13)}$ which can determine how long the subject has concentrated from his respiratory rate. The concentration can also be estimated from GSR and eye blinks as described in Sect. 2.1. However, we considered that glasses-type devices such as JINS MEME might be slightly uncomfortable for subjects who do not usually wear glasses. We believe that Spire is a more useful device because it can be easily attached to clothes and can minimize the effects on subjects during their task.

Spire is clipped on the front of the pants as shown in Fig. 2 and worn so that it is closely attached to the abdomen for accurate measurement. A previous work of Moraveji ${ }^{(14)}$ showed that Spire measures the respiration rate per minute and determines the condition on the basis of 12 to 18 breaths, which are considered to be the average respiration rate per minute for adults. Moraveji explained that Spire determines a person's state on the basis of three measures, namely, "Calmness", "Concentration", and "Tension", and the respiratory rates for these states in his research are listed in Table 1. We also estimate the concentration state in accordance with Table 1.

\subsection{Evaluation of concentration state}

In this evaluation, we confirmed the strength of each correlation in order to verify the relationships between the number of tasks and the concentration state, and the work time and

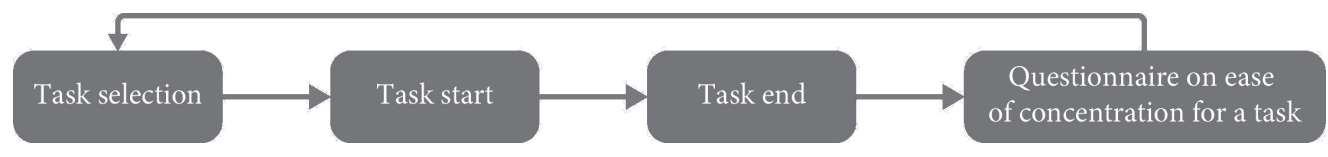

Fig. 1. Transition diagram of task recording application. 


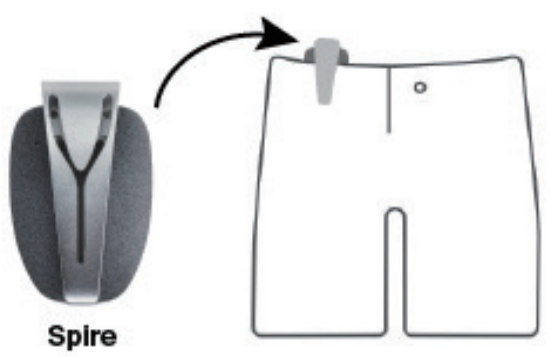

Fig. 2. Installation location of Spire.
Table 1

State and respiratory rate determined by Spire.

\begin{tabular}{lcc}
\hline State & $\begin{array}{c}\text { Respiratory rate } \\
\text { (times } / \text { min) }\end{array}$ & Breathing state \\
\hline Calmness & 6 to 12 & $\begin{array}{c}\text { Slow breathing } \\
\text { Consistent } \\
\text { breathing }\end{array}$ \\
Concentration & 16 to 20 & $\begin{array}{c}\text { Unstable and fast } \\
\text { breathing }\end{array}$ \\
\hline
\end{tabular}

the concentration state, which is the purpose of the experiment.

$$
r_{x y}=\frac{\sum_{i=1}^{n}\left(x_{i}-\bar{x}\right)\left(y_{i}-\bar{y}\right)}{\sqrt{\sum_{i=1}^{n}\left(x_{i}-\bar{x}\right)^{2}} \sqrt{\sum_{i=1}^{n}\left(y_{i}-\bar{y}\right)^{2}}}
$$

We use the Pearson correlation coefficient shown in Eq. (1) to measure the correlation strength. ${ }^{(15)}$ " $n$ " in Eq. (1) is the number of data values. Equation (1) takes values from -1.0 to 1.0 , where a negative value indicates a negative correlation and a positive value a positive correlation. Additionally, Pearson's product coefficient correlation coefficient is a scale with an absolute value from 0 to 1.0. The strength of correlation is based on the Guildford criteria shown in Table 2. ${ }^{(16)}$

\section{Results}

This section describes the results of the experiment.

\subsection{Relationship between number of tasks and concentration state in each task}

Here, we describe the results for the number of tasks and the task time, the determination of the concentration state in each task, and the correlation between the number of tasks and the total work time when Spire indicates a state of concentration.

\subsubsection{Number of tasks and task time}

Table 3 shows the number of tasks of each task type over 5 days for all 4 subjects in this experiment. In addition, "going out" was excluded from the work because none of the subjects went out.

The time required for each task was determined by the task recording application to be different from the actual time. Therefore, we compared the motion records of all subjects taken during the experiment with the work records and corrected for the occurrence of interruptions and the timing of switching tasks. Next, the proportion of time that all subjects worked on each 
Table 2

Interpretation of correlation coefficients based on Guildford criteria.

\begin{tabular}{lc}
\hline \multicolumn{2}{c}{ Size of correlation interpretation } \\
\hline 0.90 to $1.00(-0.90$ to -1.00$)$ & Very high positive (negative) correlation \\
0.70 to $0.90(-0.70$ to -0.90$)$ & High positive (negative) correlation \\
0.50 to $0.70(-0.50$ to -0.70$)$ & Moderate positive (negative) correlation \\
0.30 to $0.50(-0.30$ to -0.50$)$ & Low positive (negative) correlation \\
0.00 to $0.30(-0.00$ to -0.30$)$ & Little if any correlation \\
\hline
\end{tabular}

Table 3

Number of tasks for each task type (5 days).

\begin{tabular}{lccccc}
\hline & Subject A & Subject B & Subject C & Subject D & Total of each task type \\
\hline Document preparation & 61 & 114 & 68 & 91 & 334 \\
Document perusal & 15 & 21 & 38 & 31 & 105 \\
What to investigate & 7 & 17 & 2 & 14 & 40 \\
Email correspondence & 8 & 26 & 2 & 30 & 66 \\
Meeting & 7 & 18 & 28 & 9 & 62 \\
Telephone call & 11 & 23 & 28 & 12 & 74 \\
Going out & 31 & 44 & 33 & 39 & 147 \\
Resting, thinking & 48 & 51 & 31 & 80 & 210 \\
Total number of tasks & 188 & 314 & 230 & 306 & 1038 \\
\hline
\end{tabular}

task was calculated. Figure 3 shows the results for "document preparation" and "meeting". The percentage of tasks consuming less than $10 \mathrm{~min}$ was high and accounted for over $60 \%$.

\subsubsection{Determination of concentration state in each task}

As a result of determining the concentration state during all tasks of all subjects using Spire, it was found that there were tasks in which the concentration state was indeed for 1 min or more and tasks in which the concentration state was not induced at all. The results are shown in Table 4, where $\mathrm{T}$ indicates that the concentration state induced for $1 \mathrm{~min}$ or more was observed over 5 days, and $\mathrm{F}$ is a task in which the concentration state induced for 1 min was not observed over 5 days. Only the "document preparation" and "document perusal" tasks induced the concentration state in all subjects, while no other tasks induced the concentration state in all subjects. Therefore, we will focus on "document preparation" and "document perusal" for further detailed discussions. Also, we will discuss "all tasks" including them.

Table 5 shows the total time in the concentration state for each subject determined using Spire. Table 5 shows the following.

- The longest time in the concentration state was $92 \mathrm{~min}$ on the first day for subject $\mathrm{C}$.

- The time in the concentration state was closest to $3 \mathrm{~min}$ on the second day for subject $\mathrm{B}$.

- For subjects $\mathrm{A}$ to $\mathrm{C}$, there were days when the time in the concentration state was less than $20 \mathrm{~min}$, while for subject $\mathrm{D}$, the time in the concentration state was $40 \mathrm{~min}$ or more on all five days. 


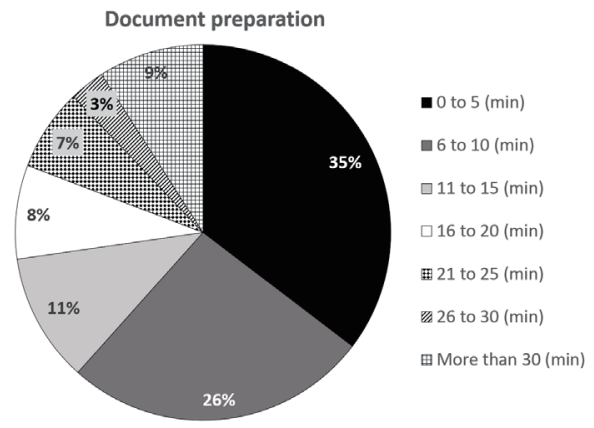

(a)

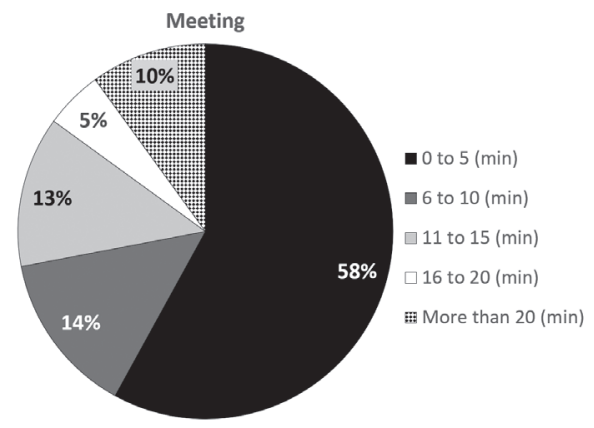

(b)

Fig. 3. Percentage of time spent on (a) "document preparation" and (b) "meeting".

Table 4

Tasks judged to induce concentration for one minute or more.

\begin{tabular}{lcccc}
\hline & Subject A & Subject B & Subject C & Subject D \\
\hline Document preparation & $\mathrm{T}$ & $\mathrm{T}$ & $\mathrm{T}$ & $\mathrm{T}$ \\
Document perusal & $\mathrm{T}$ & $\mathrm{T}$ & $\mathrm{T}$ & $\mathrm{T}$ \\
What to investigate & $\mathrm{T}$ & $\mathrm{T}$ & $\mathrm{F}$ & $\mathrm{T}$ \\
Email correspondence & $\mathrm{F}$ & $\mathrm{T}$ & $\mathrm{T}$ & $\mathrm{F}$ \\
Meeting & $\mathrm{F}$ & $\mathrm{T}$ & $\mathrm{T}$ & $\mathrm{T}$ \\
Telephone call & $\mathrm{F}$ & $\mathrm{F}$ & $\mathrm{T}$ & $\mathrm{T}$ \\
Away & $\mathrm{T}$ & $\mathrm{F}$ & $\mathrm{F}$ & $\mathrm{T}$ \\
\hline
\end{tabular}

Table 5

Total time in the concentration state for each subject.

\begin{tabular}{lccccc}
\hline & Day 1 & Day 2 & Day 3 & Day 4 & Day 5 \\
\hline Subject A & 54 & 30 & 19 & 58 & 77 \\
Subject B & 16 & 3 & 23 & 34 & 25 \\
Subject C & 92 & 17 & 79 & 37 & 48 \\
Subject D & 47 & 51 & 47 & 72 & 48 \\
\hline
\end{tabular}

\subsubsection{Correlation between the number of tasks and total concentration time as determined using Spire}

In order to verify the relationship between the number of tasks and the concentration state, we calculated the correlation coefficient between the number of tasks for each subject per day and the total concentration time determined using Spire for each subject. The correlation coefficient is $-0.445(p<0.05)$, which indicates a low negative correlation on the basis of Gilford's criteria.

Figure 4 shows the number of tasks per day for all subjects and the total concentration time determined by Spire for each subject. When the number of tasks per day is large, the concentration time becomes short, and when the number of tasks is small, the total concentration time tends to be long.

\subsection{Relationship between concentration state and work time}

We examine the relationship between the work time until the subjects begin to be concentrate and the task type. At the time of examination, we defined and compared tasks as follows. 


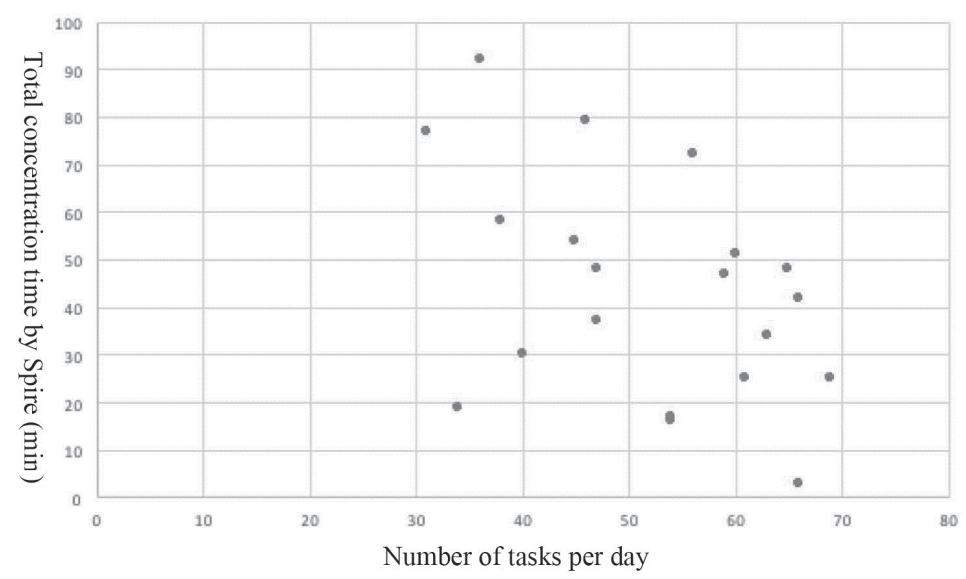

Fig. 4. Number of tasks and total concentration time per day.

- In-concentration-state tasks: tasks for which the concentration state lasting at least $1 \mathrm{~min}$ was confirmed.

- In-nonconcentration-state tasks: tasks for which the concentration state lasting more than 1 min was not confirmed.

The tasks to be examined are "all tasks", "document preparation," and "document perusal" presented in Sect. 5.1.2. In addition, the effects of interruptions were considered by analysis with and without interruptions. The results are shown in Table 6. There is a significant difference between the work times of the concentrated and nonconcentrated tasks except for "document perusal".

Tables 7 and 8 show the average work times. Their features are described below.

[With interruptions]

- The average work time of the concentrated task for "all tasks", "document preparation", and "document perusal" is longer than that of the nonconcentrated task

- The average work time of the concentrated task is more than twice that of the nonconcentrated task

[Without interruptions]

- The average work time of the concentrated task for "all tasks", "document preparation", and "document perusal" is longer than that of the nonconcentrated task.

- The average work time is shorter than those with interruptions regardless of the task type.

From the above results, it can be seen that the concentrated task took longer than the nonconcentrated task regardless of the occurrence or absence of interruptions. This indicates that there is little work being done in a concentration state in a short work time. Therefore, in order to perform tasks in the concentration state, it is considered necessary to secure tasks with 
Table 6

Concentrated/nonconcentrated task and work time examination results.

\begin{tabular}{lc}
\hline Examination of work time for concentrated/nonconcentrated task (with interruptions) \\
\hline All tasks & Significant difference $(p<0.05)$ \\
Document preparation & Significant difference $(p<0.05)$ \\
Document perusal & No significant difference $(p>0.05)$ \\
\hline Examination of work time for concentrated/nonconcentrated task (without interruptions) \\
\hline All tasks & Significant difference $(p<0.05)$ \\
Document preparation & Significant difference $(p<0.05)$ \\
Document perusal & Significant difference $(p<0.05)$ \\
\hline
\end{tabular}

Table 7

Average working hours (with interruptions) [min].

\begin{tabular}{lcc}
\hline Average work time (min) & Concentrated task & Nonconcentrated task \\
\hline All tasks & 22.3 & 7.95 \\
Document preparation & 25.5 & 11.1 \\
Document perusal & 21.0 & 10.3 \\
\hline
\end{tabular}

Table 8

Average working hours (without interruptions) [min].

\begin{tabular}{lcc}
\hline Average work time (min) & Concentrated task & Nonconcentrated task \\
\hline All tasks & 19.6 & 6.96 \\
Document preparation & 22.1 & 8.35 \\
Document perusal & 16.4 & 6.07 \\
\hline
\end{tabular}

a certain number of working hours. In particular, it is considered necessary to continue the work for more than $8 \mathrm{~min}$ in "document preparation" and for more than $6 \mathrm{~min}$ in "document perusal".

\subsection{Examination by concentration ratio}

Next, we focus on the concentration ratio and verify the concentrated task and work time. The concentration ratio is the ratio of the actual time in minutes for an in-concentration-state task per work hour, as shown in Eq. (2). In order to verify the relationship between the work time and the concentration ratio, we calculated the correlation coefficient between the work time and concentration ratio of the concentrated task. In addition, considering interruptions, examination was performed with and without interruptions.

$$
\text { Concentration ratio }=\frac{\text { Actual time for in-concentration-statetask }(\mathrm{min})}{\text { Work hour }}
$$

\subsubsection{Correlation between concentration ratio and work time (with interruptions)}

Table 9 shows the correlation coefficient between the work time and the concentration ratio when interruptions occurred in the concentrated work. In addition, the "document perusal" was 
Table 9

Correlation coefficient between concentrated task (with interruptions) and concentration ratio.

\begin{tabular}{lcc}
\hline & All tasks & Document preparation \\
\hline All subjects & -0.438 & -0.623 \\
$p$-value & $p<0.05$ & $p<0.05$ \\
\hline
\end{tabular}

omitted because the number of data was insufficient. On the basis of the Guildford criteria, "all tasks" confirmed a low negative correlation. "Document preparation" showed little if any correlation. In other words, there is a correlation between the concentration ratio and the work time in the concentrated task (with interruptions). Figure 5 shows the concentration ratio relative to the work time. The concentration ratio decreased as the work time increased.

\subsubsection{Correlation between concentration ratio and work time (without interruptions)}

For concentrated tasks without interruptions, the correlation coefficient between the work time and the concentration ratio was as described in Sect. 5.3.1. The results are shown in Table 10. On the basis of the Guildford criteria, "all tasks" and "document preparation" showed a low negative correlation. "Document perusal" showed a moderate negative correlation. In other words, it was found that there is a correlation between the concentration ratio and the work time even without interruptions. Figure 6 shows the concentration ratio relative to the number of business hours. As a result, it was found that the concentration ratio decreased as the work time increased, as in the case with interruptions.

\subsection{Relationship of work times obtained with and without interruptions}

In the concentrated task, we compared and examined the work times obtained with and without interruptions. The results are shown in Table 11. No significant difference in work time was found between concentrated tasks with or without interruptions.

\section{Discussion}

In this section, we discuss the experimental results.

\subsection{Relationship between concentration state and number of tasks}

As described in Sect. 5.1, the correlation coefficient calculated from the number of tasks per day of each subject and the concentration time in each task showed a moderate negative correlation on the basis of the Guildford criteria. From this result, we found that when the number of tasks per day increases, the total concentration time tends to decrease. Therefore, it is considered better to continue one task to some extent in order to improve the task efficiency, focusing on the concentration state. 
All tasks

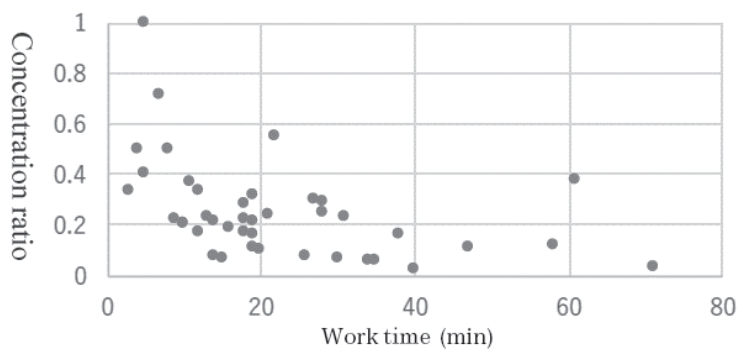

(a)
Document preparation

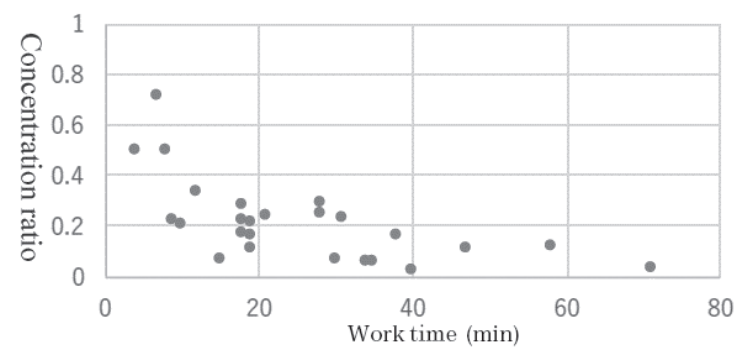

(b)

Fig. 5. Work time vs concentration ratio (with interruptions).

Table 10

Correlation coefficient between concentrated task (without interruptions) and concentration ratio.

\begin{tabular}{lccc}
\hline & All tasks & Document preparation & Document perusal \\
\hline All subjects & -0.428 & -0.424 & -0.553 \\
$p$-value & $p<0.05$ & $p<0.05$ & $p<0.05$ \\
\hline
\end{tabular}

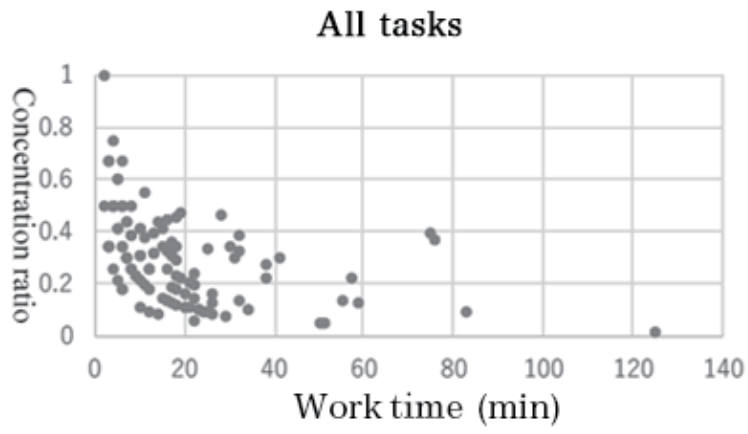

(a)

Document preparation

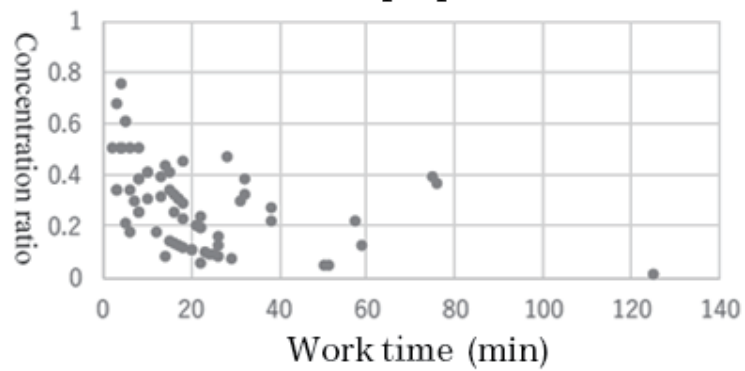

(b)
Document perusal

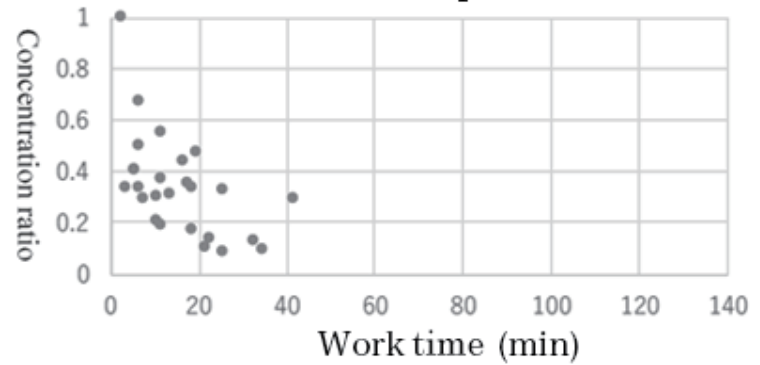

(c)

Fig. 6. Graphs of work times obtained and concentration ratio (without interruptions).

Table 11

Examination result of work hours with and without interruption.

\begin{tabular}{lc}
\hline All tasks & Document preparation \\
\hline Document preparation & No significant difference $(p>0.05)$ \\
Document perusal & No significant difference $(p>0.05)$ \\
\hline
\end{tabular}




\subsection{Relationship between concentration state and work time}

As explained in Sect. 5.2, concentration could not be confirmed if the work time was short, and it was necessary to continue the task for a certain period of time to achieve the concentration state. Specifically, we found that the concentration state cannot be confirmed, that is, the time of the nonconcentrated task was determined to be on average 11 min for "document preparation", 10 min for "document perusal". Therefore, we found that it is necessary to carry out tasks longer than this time to achieve the concentration state.

On the other hand, when analyzing the relationship between the concentration state and the work time focusing on the concentration ratio, as described in Sect. 5.3, the concentration ratio decreases as the work hour increases. Therefore, we considered that the longer the time, the less efficient the work.

In addition, Fig. 7 shows the time from the start of the concentrated task to the time of the concentration state onset. The horizontal axis shows the time from the start of the task and the vertical axis shows the time of the concentration state onset. We can see from Fig. 7 that there are many cases where concentration starts within about 10 min from the start of the task. On the other hand, after about 10 min from the start of the task, there are extremely few cases in which concentration starts. In other words, if $10 \mathrm{~min}$ has passed since the start of the task and the subject has not reached the concentration state by that time, it will be difficult to attain the concentration state even if the task is continued further.

From the above results, we found that, to carry out desk work efficiently, if the concentration state sets in within $10 \mathrm{~min}$, it is advantageous to continue the task; otherwise, it is beneficial to discontinue it.

\subsection{Consideration of relationship between concentration state and degree of interruption}

As shown in Sect. 5.4, no significant difference in concentration time was found between cases with and without interruptions. Therefore, even if an interruption occurs, it is considered to not affect the work time of the concentrated task.

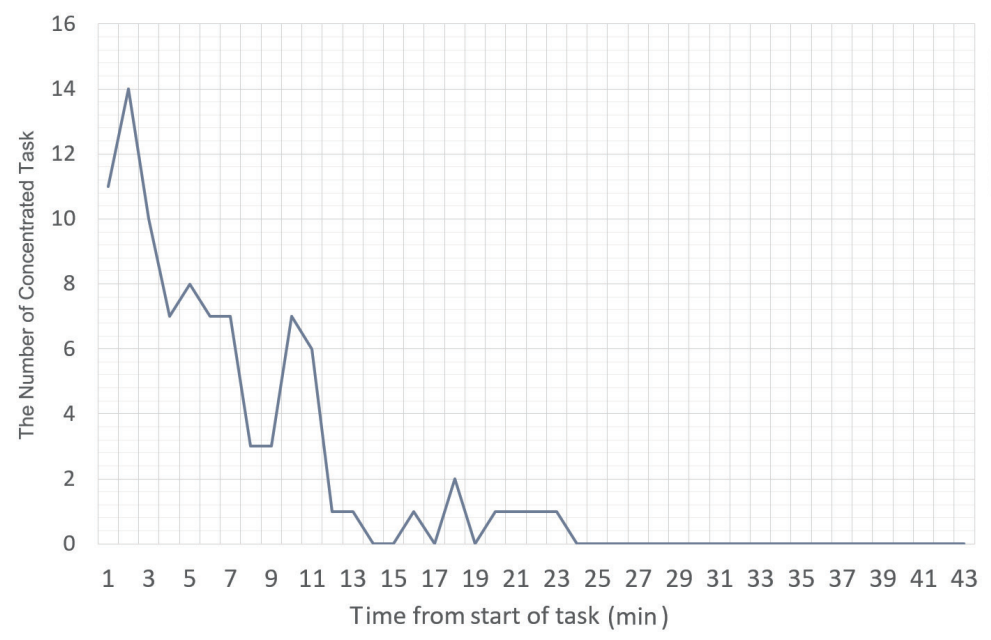

Fig. 7. Time from the start of task to time of concentration state onset. 


\section{Conclusion}

In this research, in order to improve desk work efficiency, we focused on concentration and aimed to clarify the relationship between the concentration state and the work time. In the experiment, task contents were acquired from workers actually engaged in desk work, and the concentration state of each worker was acquired using Spire. As a result, three things became clear.

The first is that the total concentration time tends to be shorter if the number of tasks per day is large. Therefore, in order to optimize operations by focusing on concentration, it is better to have a smaller number of tasks per day.

The second is that for a single task, if the task time is short, the degree concentration will be low and if the task time is long, the proportion of concentration time will be low. In the environment of this experiment, it was found that the efficiency can be improved by performing a task for $10 \mathrm{~min}$ or more. However, it was also found that if concentration was not attained within 10 min from the start of the task, it was difficult to attain concentration at a later time, so the task should be discontinued.

The third is that an interruption did not affect the concentrated task.

After the experiment, we did some interviews and shared knowledge with Toyama prefectural government officials. They responded that they would pay more attention to their workload if workload had impact on the concentration state. In addition, they requested us to provide useful information to determine their time allocation for the work. However, they placed importance on making their own schedule, not the schedule that the computer decides. Therefore, as a future perspective, we will present the optimum work time to workers focusing on the concentration state that was clarified in this study and evaluate its effects. In particular, there will be a need for a system that dynamically determines whether to continue tasks when concentration is observed within 10 min of the start of the task.

In addition, to further discuss desk work efficiency, we also consider information presentation methods. For example, at an actual site, priority can be given to the completion of a task, and the task can be continued even after a rest. Therefore, we will advance research on the basis of persuasive technology ${ }^{(17,18)}$ as a way to persuade workers to discontinue tasks.

In this study, we conducted experiments in a normal season. On the other hand, we found that it was necessary to conduct further research and measure experiments conducted at different work statuses of subjects.

Furthermore, in this experiment, we did not consider the parallel processing of work in the environment. However, desk work is diverse and parallel processing may occur. In that case, we must investigate the influence of concurrent task on the concentration state when working a task during another task. If this does not affect, we may consider parallel processing (two tasks) as one side task. Otherwise, we must redefine the combination of tasks as a single task. For example, we must define "create a mail during a meeting" as the single task "meeting + mail correspondence".

Since the prefectural officer generally rotates various jobs every three years, we found that there were differences between their first year and third year experiences from interviews. For this reason, we expect that there will be a difference in the degree of concentration due 
to a difference in the number of years of service such as in the cases of experienced and new employees. In addition, we learned from interviews that the time during which people could concentrate might differ individually. Therefore, we need to consider personal attributes such as the number of years of service in future research.

In the future, this research in other industries will be extended. For example, if workers could not concentrate enough on their job at factories in manufacturing industries, they may have serious problems. Therefore, we believe that investigating the relationship between concentration and work in this study is meaningful. In this case, the knowledge gained from the experiment will lead to not only operational efficiency but also risk management.

\section{References}

1 Ministry of Health Labour and Welfare: https://www.mhlw.go.jp/english/policy/employ-labour/labourstandards/dl/201904kizyun.pdf (accessed August 2019).

2 The Japan Institute for Labour Policy and Training: https://www.jil.go.jp/kokunai/statistics/databook/2017/03/ p107-114_t3-4.pdf (accessed August 2019).

3 M. Okubo and A. Fujimura: Int. Conf. Human-Computer Interaction (HCI, 2009) 131-140. https://doi. org/10.1007/978-3-642-02574-7_15

4 A. Z. H. Shukor, N. A. Natrah, M. A. Afiq, M. H. Jamaluddin, Z. A. Ghani, H. N. M. Shah, and M. Z. Ab Rashid: J. Telecommun. Electron. Comput. Eng. (JTEC) 10 (2018) 53.

5 S. Honda, H. Tomioka, T. Kimura, T. Oosawa, K. I. Okada, and Y. Matsushita: Inf. Software Technol. 41 (1999) 383. https://doi.org/10.1016/S0950-5849(98)00070-6

6 K. Yajima, R. Iwatsuki, and S. Takahashi: Procedia Comput. Sci. 96 (2016) 1447. https://doi.org/10.1016/ j.procs.2016.08.190

7 Y. Uema and K. Inoue: Proc. 2017 ACM Int. Joint Conf. Pervasive and Ubiquitous Computing and Proceedings of the 2017 ACM Int. Symp. Wearable Computers (ACM, 2017) 297-300. https://doi. org/10.1145/3123024.3123189

8 S. Hashimoto, T. Tanaka, K. Aoki, and K. Fujita: Int. Conf. Human Interface and the Management of Information (HIMI, 2013) 297-306. https://doi.org/10.1007/978-3-642-39226-9_33

9 G. Mark, D. Gudith, and U. Klocke: Proc. SIGCHI Conf. Human Factors in Computing Systems (2008) 107110. https://doi.org/10.1145/1357054.1357072

10 F. B. Benatti and M. Ried-Larsen: Med. Sci. Sports Exercise (MSSE) 47 (2015) 2053. https://doi.org/10.1249/ MSS.0000000000000654

11 K. De Cocker, I. De Bourdeaudhuij, G. Cardon, and C. Vandelanotte: BMC Public Health 15 (2015) 959 https:// doi.org/10.1186/s12889-015-2288-y

12 E. Vlemincx, J. Taelman, S. De Peuter, I. Van Diest, and O. Van Den Bergh: Psychophysiology 48 (2011) 117. https://oi.org/10.1111/j.1469-8986.2010.01043.x

13 Spire: https://spire.io (accessed August 2019).

14 N. Moraveji: The Science Behind Spire. http://parkinsoncenter.org/wp-content/uploads/Science-Behind-Spire. pdf (accessed August 2019).

15 J. Benesty, J. Chen, Y. Huang, and I. Cohen: Noise Reduction in Speech Processing (2009) 1-4. https://doi. org/10.1007/978-3-642-00296-0_5

16 J. P. Guilford: Fundamental Statistics in Psychology and Education (1950). https://psycnet.apa.org/ record/1950-04974-000 (accessed August 2019).

17 B. J. Fogg: Ubiquity (2002) 5. https://doi.org/ 10.1145/764008.763957

18 H. Oinas-Kukkonen: 5th Int. Conf. Persuasive Technology (PERSUASIVE, 2010) 4-14. https://doi. org/10.1007/978-3-642-13226-1_3 


\section{About the Authors}

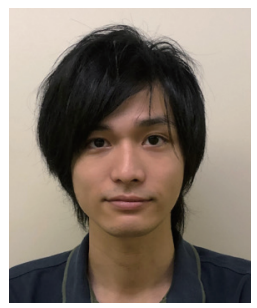

Shinnosuke Date received his M.S. degree from Toyama Prefectural University, Japan, in 2018. Since 2018, he has been in the doctoral course of Toyama Prefectural University. His research interests include behavioral changes and ubiquitous computing. (s-date@puc.pu-toyama.ac.jp)

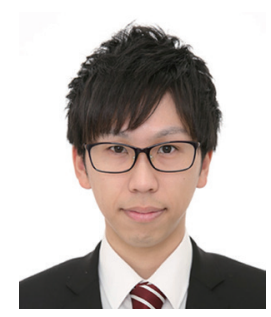

Yuta Miyake received his M.S. degree from Toyama Prefectural University, Japan, in 2019. In 2019, he joined DOCOMO Technology, Inc. His research interest at Toyama Prefectural University was ubiquitous computing.

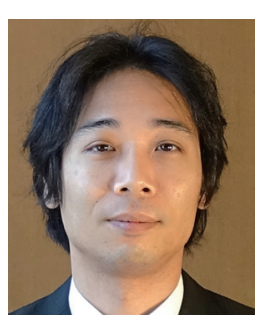

Takeshi Iwamoto received his Ph.D. in media and governance from Keio University. After postdoctoral research at the KDDI Laboratory, Japan, he joined Toyama Prefectural University, Japan. He mainly works on research concerning application areas of smart sensing, Internet of Things, and activity recognition. (iwamoto@pu-toyama.ac.jp) 